\title{
Identificação das necessidades de interação dos usuários em sistemas ERP: proposta de uma metodologia investigativa
}

\author{
Joelma Choma ${ }^{1}$, Luciana A. M. Zaina ${ }^{1}$, Adriana M. Amaral ${ }^{2}$, Pedro R. Oliveira ${ }^{2}$ \\ ${ }^{1}$ Universidade Federal de São Carlos - Campus Sorocaba (UFSCar-Sorocaba) \\ Sorocaba - SP - Brasil \\ ${ }^{2}$ Mega Sistemas Corporativos \\ Itu - SP - Brasil \\ \{jhchoma, lzaina\}@ufscar.br, \{adriana.amaral, pedro\}@mega.com.br
}

\begin{abstract}
This article discusses a research methodology for detecting techniques of data collection for identification of user needs, an integral part of the search for efficient mechanisms for the development of ERP systems that allow for better user interaction and contribute in various stages of the process development. The proposed methodology has been validated with an experiment conducted in Brazilian industry developer of ERP systems, whose results are presented in the report of the case study.
\end{abstract}

Resumo. Este artigo trata sobre uma metodologia investigativa para detectar técnicas de coleta de dados para identificação das necessidades dos usuários, parte integrante da busca por mecanismos eficientes para o desenvolvimento de sistemas ERP que permitam uma melhor interação com o usuário e contribuam em várias etapas do processo de desenvolvimento. A metodologia proposta foi validada com um experimento realizado em indústria brasileira desenvolvedora de sistemas ERP, cujos resultados são apresentados no relato do estudo de caso.

\section{Introdução}

É inquestionável o fato de que sistemas ERP (Enterprise Resource Planning - Sistema de Gestão Empresarial Integrado) trouxeram grandes vantagens ao meio corporativo otimizando o fluxo de informações e acesso aos dados operacionais, integrando diversos setores da empresa, viabilizando diferentes processos de negócios, enxugando a estrutura organizacional e tornando-a mais flexível.

Os sistemas ERP evoluíram a partir dos anos 90, sempre com uma forte preocupação com o foco funcional, para acompanhar as novas tecnologias disponíveis e constantemente se adequar às imposições fiscais e necessidades mercadológicas [Padilha e Marins 2005], [Scott 2008]. A complexidade inerente desse tipo de software colabora para que sua implantação, na grande maioria dos casos, seja considerada problemática [Nunes et al. 2012], e que exija muito esforço dos usuários na aprendizagem e uso, fato que se agrava pelo fraco design de interação característico desses sistemas. Consequentemente, a qualidade de utilização em sistemas ERP é limitada e reduz a eficiência no trabalho devido à alta curva de aprendizado para que o usuário reconheça e compreenda todas as funcionalidades da tecnologia [Chou et al. 2014].

Uflacker e Busse [2007] acreditam que a quantidade de complexidade percebida pode ser reduzida por um design de interação focado em apoiar o fluxo de trabalho do 
usuário. Problemas referentes à interação são comuns dentro do domínio de sistemas ERP e grande parte dos problemas de design é atribuída a pouca comunicação existente com usuário final com o intuito de explorar suas reais necessidades.

Normalmente, no domínio de desenvolvimento de sistemas ERP as interfaces com o usuário são elaboradas por desenvolvedores com visões funcionais, sem um eficiente levantamento exploratório dos requisitos de interação do usuário junto ao público alvo.

Neste contexto surge a motivação pela investigação de metodologias de design de interação e práticas de experiência do usuário (user experience) para serem incorporadas nos processos de desenvolvimento de softwares ERP que atualmente estão voltados ao modelo funcional. Nesse sentido a área de IHC (Interação Humano-Computador) contribui com diversas técnicas que ao longo do tempo foram desenvolvidas e experimentadas por especialistas da área, as quais podem ser selecionadas e inseridas de forma eficaz no processo de desenvolvimento de sistemas ERP. O presente artigo descreve a proposta de uma metodologia investigativa utilizada para selecionar as melhores técnicas de coleta de dados para identificação das necessidades de interação dos usuários de sistemas ERP. O objetivo principal é que a equipe de desenvolvimento, com seus diferentes papéis, vivenciem as necessidades de interação do usuário e possa escolher as técnicas de maneira que estas sejam adequadas ao público alvo em questão.

O artigo está estruturado da seguinte forma: na Seção 2 são apresentados os fundamentos teóricos e trabalhos relacionados à proposta; a Seção 3 descreve a metodologia investigativa sobre técnicas de coleta para sistemas ERP; a Seção 4 relata o estudo de caso para validação da proposta e análise dos resultados obtidos; e por fim a Seção 5 discorre sobre as considerações finais.

\section{Fundamentos teóricos e trabalhos relacionados}

Trabalhar conscientemente para a produção de sistemas que sejam altamente utilizáveis e atendam às expectativas e necessidades de seus usuários reais é a maneira de desenvolver o Design Centrado no Usuário (UCD - User-Centered Design)[Seffah et al. 2005]. Uma etapa fundamental do UCD é a pesquisa das necessidades de interação do usuário em consonância com os requisitos funcionais do sistema. Porém, os sistemas ERP acabam concentrando-se nas questões funcionais, já que normalmente há pouco contato com os usuários finais [Matthews 2008].

As técnicas de coleta de dados mais utilizadas na pesquisa com o usuário são entrevista, brainstorming, questionário, grupos em foco, etnografia pura e participativa, investigação contextual e card sorting [Courage e Baxter 2005], [Rogers et al. 2013]. A Tabela 1 apresenta de forma sucinta as técnicas.

As técnicas de investigação e observação podem ser conduzidas combinadas ao uso de artefatos de apoio, tais como protótipos de diferentes níveis de detalhes e fidelidade, cenários de uso, sketches e storyboards. Esses artefatos representam parte de uma solução de IHC que servem de insumo para uma avaliação progressiva, realizada ao longo de todo o processo de design para compreender e confirmar a compreensão sobre o que os usuários querem e precisam, e para verificar se a solução atende às necessidades dos usuários [Courage e Baxter 2005].

Para que o investigador que irá coletar os dados tenha uma visão das pessoas 
Tabela 1. Técnicas de coleta de dados

\begin{tabular}{|c|c|c|}
\hline \multicolumn{2}{|r|}{ Técnica } & Caracteristicas gerais \\
\hline \multirow{3}{*}{ 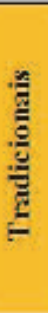 } & Entrevista & $\begin{array}{l}\text { São a dequadas para estudos exploratónos, onde não se sabe bem o que se está } \\
\text { procurando, ja que o entrevistador pode ajustar a entrevista conforme a } \\
\text { situação. }\end{array}$ \\
\hline & Brainstorming & $\begin{array}{l}\text { Busca levantar de forma bastante livre um conjunto grande e abrangente de } \\
\text { opiniões dos participantes em tomo de um tema, que resulta numa lista } \\
\text { priorizada de necessidades e desejo dos usuános. }\end{array}$ \\
\hline & Questionário & $\begin{array}{l}\text { Formulário com uma série ordenada de perguntas a serem respondidas sem a } \\
\text { interação com o pesquisador. }\end{array}$ \\
\hline \multirow{3}{*}{$\frac{\sqrt{2}}{\frac{3}{2}}$} & $\begin{array}{l}\text { Grupo em foco } \\
\text { (focus group) }\end{array}$ & $\begin{array}{l}\text { Cerca de seis a nove usuários são levados a se reunirem para discutir novos } \\
\text { conceitos e identificar problemas durante um periodo de cerca de duas horas. } \\
\text { Cada grupo é genido por um modera dor que é responsável por mantero foco do } \\
\text { grupo nas questões de interesse. }\end{array}$ \\
\hline & Etmografia & $\begin{array}{l}\text { Permite ao pesquisador observar e entender o comportamento natural de } \\
\text { usuanos enquanto desempenham uma atividade em seu próprio ambiente de } \\
\text { atuação. Etnografia pura: sem a interação do observador com os participantes. } \\
\text { Etnografia participativa: o observador pode adotar diferentes niveis de } \\
\text { participação de maneira fomal ou informal. }\end{array}$ \\
\hline & $\begin{array}{l}\text { Investigação } \\
\text { contextual }\end{array}$ & $\begin{array}{l}\text { Estudo de campo com envolvimento intenso do investigador que adota um } \\
\text { modelo de mestre-aprendiz exercendo o papel de aprendiz do trabalho do } \\
\text { usuário e o usuário no papel de mestre ensinado seu trabalho para o } \\
\text { investigador-aprendiz. }\end{array}$ \\
\hline 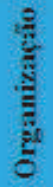 & Card sorting & $\begin{array}{l}\text { Busca encontrar pa drões na forma como os usuános organizam suasideias no } \\
\text { momento da interação e como ele entende o uso dos termos. }\end{array}$ \\
\hline
\end{tabular}

envolvidas no processo de interação utiliza-se a técnica de persona como apoio. Uma persona é um personagem fictício, modelo hipotético de um grupo de usuários reais, criado para descrever um usuário típico. Pode-se criar pelo menos uma persona por tipo de usuário. A ideia de projetar para um pequeno conjunto de personas, segundo Courage e Baxter [2005] vai garantir um maior sucesso em criar um design focado nas necessidades específicas de cada persona.

Diferentes tipos de dados resultam da coleta de dados junto ao usuário, e conforme sua classificação permitem análises quantitativa e/ou qualitativa. No caso de técnicas de observação (etnografia pura, etnografia participativa e investigação contextual) os dados brutos usuais são oriundos de anotações de observações anotadas pelos observadores, fotografias, gravações de audio e vídeo, log de dados ou comentários de conversas dos usuários. Em análise qualitativa identificar padrões recorrentes e temas, categorizar dados e analisar os incidentes críticos são tipos mais simples de executar a análise, porém pode-se estruturar a análise de dados qualitativos através de frameworks téoricos que permitem maior aprofundamento na pesquisa. Exemplos de frameworks teóricos que são frequentemente utilizados no design da interação e para análise de dados de observação são a teoria fundamentada nos dados (grounded theory) [Glaser 1992], a cognição distribuída e a teoria da atividade [Rogers et al. 2013].

O estudo realizado por Iivari [2006] mostra que compreender usuários e os seus trabalhos e tarefas é a base necessária para projetar sistemas interativos com boa usabilidade, e que sejam adequados às reais necessidades das pessoas. Existem diferentes pontos 
de vista sobre como envolver os usuários no processo de design, sendo que o envolvimento pode ser informativo e consultivo quando os usuários agem apenas como provedores de informação, objetos de observação ou comentaristas de soluções de design predefinidos; ou participativo quando são agentes ativos no processo de design e têm poder de decisão. Iivari [2006] sugere, no entanto, que o contexto cultural da organização seja entendido primeiro em profundidade, para depois adotar a abordagem mais adequada.

Incorporar elementos de interação que estimulem e que favoreçam a percepção do usuário, mecanismos de apoio à realização das tarefas complexas, identificação da localização do usuário dentro do sistema, mensagens de retorno adequadas ao usuário final, navegação com estruturas mais simples, entre outros, são fatores que aparecem para solucionar os maiores problemas encontrados em sistemas ERP. Os estudos de Faisal et al. [2012] e Singh e Wesson [2009] demonstram que há a necessidade de identificar e compreender os problemas de design da interação através de um conjunto de critérios específicos para sistemas ERP.

Marshall et al. [2013] confirmam que personas e conjuntos de dados de usuários são metodologias complementares usadas para identificar as necessidades dos usuários. As personas servem para compreensão e visualização de objetivos do usuário, motivações, relações com produtos e contextos de uso existentes, ter um enfoque psicológico e são usadas para definir o que os usuários querem ganhar com a utilização de um produto ou serviço. Além disso, são importantes ferramentas de comunicação para assegurar que todos da equipe de desenvolvimento tenham a mesma compreensão sobre as necessidades do usuário. Os conjuntos de dados coletados servem para prover dados técnicos aplicáveis, associados com conjuntos de dados de usuários e para fornecer dados de atitude associados com personas.

\section{Metodologia investigativa proposta}

Observa-se que embora a coleta ocorra no desenvolvimento de sistemas ERP estas muitas vezes são feitas sem planejamento do que se deseja investigar. Com o objetivo de introduzir um mecanismo que propiciasse aos desenvolvedores de sistemas ERP a escolha da melhor técnica de coleta a ser utilizada para investigação das necessidades de interação do usuário foi proposta uma metodologia. Com a metodologia busca-se não só introduzir a cultura do uso dos métodos de pesquisa das necessidades do usuário bem como introduzir artefatos que apoiem a coleta com foco na experiência do usuário [Schylström 2013].

A metodologia proposta é composta de cinco etapas. Sugere-se que ela seja aplicada envolvendo pessoas com diferentes papéis dentro do processo de desenvolvimento da empresa, pois o objetivo é que todos visualizem a importância do uso das técnicas de coleta nas diferentes fases de desenvolvimento. Além disto, é importante que ele seja conduzido por um profissional de UX (User Experience - Experiência do Usuário) com vivência de uso das diferentes técnicas. O profissional de UX faz o papel de condutor do processo e aparece em todas as fases como ponto de apoio e esclarecedor de dúvidas. A Figura 1 apresenta a metodologia proposta.

Na primeira etapa, denominada de Fundamentos da Coleta $\mathbf{x}$ UX, são apresentados fundamentos sobre UCD, um grupo de técnicas para identificação das necessidades do usuário (brainstorming, entrevistas, questionários, focus group, etnografia participativa, investigação contextual e card sorting), artefatos de apoio na coleta de dados (protótipos, 
cenários, sketches e storyboard) e uma introdução sobre personas. Durante esta etapa deve-se buscar relacionar as técnicas de coleta com a investigação das necessidades de interação.

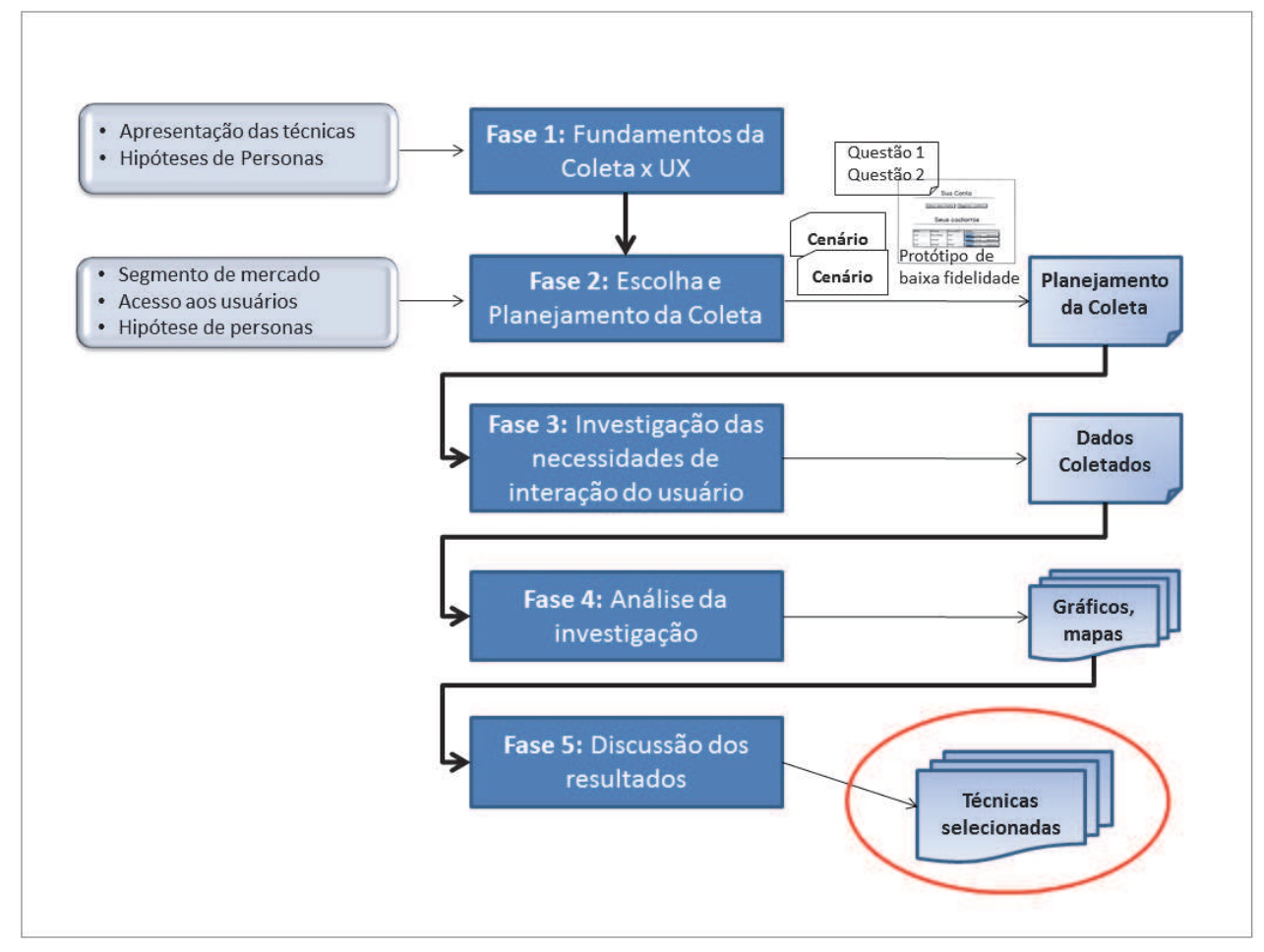

Figura 1. Metodologia proposta

Escolha e Planejamento da Coleta se constitui na segunda etapa, onde os participantes devem formar grupos que possuam membros para atuar em diferentes papéis do processo de desenvolvimento, tais como coordenador de projeto, desenvolvedor, arquiteto e testador, com a tarefa de planejarem uma atividade de coleta de dados com o foco nos usuários alvo de um determinado módulo de ERP. É importante destacar que a metodologia proposta pode ser utilizada tanto para design como para redesign do sistema. Durante esta etapa o grupo deverá realizar a escolha de uma ou mais técnicas de investigação considerando os usuários alvos (já visualizados como hipóteses de personas), o segmento de mercado que o ERP atinge e o acesso aos usuários. Neste ponto é fundamental o papel do profissional de UX condutor do workshop. Também é importante que todas as informações do planejamento sejam registradas. Devem ser informados nome e papel de cada integrante do grupo; nome dos clientes, número de usuários envolvidos na coleta e hipótese de personas; módulo do sistema que está sendo estudado e se fazem parte de um projeto de design ou redesign; o objetivo da coleta; as técnicas utilizadas e os artefatos de apoio. Os detalhamentos do que será observado ou sobre os questionamentos feitos ao usuário devem constar no planejamento. O trabalho minucioso desta etapa se constitui em um dos elementos chaves para uma coleta bem sucedida.

Na terceira etapa, Investigação das necessidades de interação do usuário, os grupos devem providenciar a coleta de dados aplicando o planejamento da etapa anterior. Embora este seja um trabalho dos grupos, o profissional de UX realiza o papel de ponto 
de apoio novamente.

A Análise da investigação ocorre na quarta etapa na qual são apresentadas as técnicas de análise de dados quantitativos e qualitativos com a finalidade de capacitar os grupos para analisar os dados coletados e sumarizar os resultados. Além disto, são apresentadas formas de organizar os dados das personas que neste ponto passam de hipóteses para personas concretas.

Na quinta e última etapa, Discussão dos resultados, os grupos apresentam e compartilham os resultados obtidos motivando a discussão entre os participantes sobre as vantagens e desvantagens de cada técnica utilizada. O resultado mais relevante da última etapa é a identificação das técnicas mais adequadas e aderentes às necessidades de desenvolvimento da empresa e do tipo de usuário.

Sistemas ERP são genéricos e atendem conjuntos diferentes de usuários por isso justifica-se a proposta de investigação para determinar as técnicas de coleta mais apropriadas para levantar necessidades com grupos distintos de usuários.

\section{Validação da metodologia: um estudo de caso}

Para validação da metodologia proposta foi realizado um workshop. O workshop "User Experience - pesquisa com o usuário" aconteceu no período de setembro a outubro de 2013, com o objetivo de apresentar inicialmente aos participantes métodos de pesquisa com o usuário final para capacitá-los para o planejamento e a aplicação de um dos métodos em usuários alvos da empresa, a fim de coletar dados para serem analisados e fornecer informações para identificar personas.

O workshop foi realizado na sede da Mega Sistemas Corporativos situada na cidade de Itu - São Paulo, empresa com 29 anos de experiência no ramo de desenvolvimento de sistemas ERP, muito atuante nos segmentos da Construção Civil, Manufatura, Logística, Combustível e Agronegócio. Atualmente, atende uma carteira de 2000 clientes com mais de 50.000 usuários e conta com 700 colaboradores.

Participaram do workshop 16 colaboradores profissionais de diferentes áreas de desenvolvimento de software, sendo 1 arquiteto de sistemas, 3 líderes técnicos, 3 analistas de sistemas, 4 testadores e 5 desenvolvedores. A maioria dos participantes (06 pessoas/ 40\%) possui mais de 10 anos de experiência na área de desenvolvimento de software e no desenvolvimento de sistemas ERP.

\subsection{Metodologia na Prática}

Na primeira etapa do workshop foi apresentado aos participantes fundamentos de UCD, técnicas, métodos e artefatos de coleta de dados e uma parte introdutória sobre hipóteses de personas. Preparados para a próxima fase os participantes foram orientados a formarem quatro grupos de quatro pessoas de forma que dentro de cada grupo haveria uma distribuição de papéis preferencialmente com um elemento de cada área. Outra questão resolvida nesta fase foi a definição de quais partes do sistema ERP seriam objetos da pesquisa de cada grupo. Foram determinados dois módulos: Solicitação de Compras e Pedido de Vendas. Quanto ao foco da coleta este poderia ser de design ou redesign. A distribuição ficou da seguinte maneira: Grupo 1 e Grupo 3 - redesign da interface do módulo de Solicitação de Compra; Grupo 2 - redesign da interface do módulo de Pedido de Vendas e Grupo 4 - design da interface do módulo de Pedido de Vendas. 
Na segunda etapa, segundo dia de workshop, os participantes trabalharam em grupos no planejamento da atividade de coleta registrando todas as informações no formulário online. A maioria dos grupos combinou mais de uma técnica na atividade de coleta e as técnica utilizadas foram a etnografia participativa (Grupos 1 e 2), investigação contextual (Grupo 1), entrevista (Grupos 2, 3 e 4) e card sorting (Grupos 1 e 3).

$\mathrm{Na}$ terceira etapa, fase de coleta, os grupos tiveram aproximadamente vinte dias para contatar o cliente e executar a atividade de coleta. Logo no início do período cada grupo recebeu como feedback um documento sumarizando a primeira parte do workshop, um resumo contendo informações do planejamento de cada grupo tais como integrantes, foco da coleta, parte do ERP, técnicas escolhidas, clientes alvo e um parecer quanto ao resultado do planejamento.

$\mathrm{Na}$ quarta etapa do workshop, os grupos tiveram a tarefa de fazer a análise dos dados coletados (quantitativa e/ou qualitativa) e validar a hipótese das personas elaboradas previamente na segunda etapa.

Como as técnicas de coleta escolhidas pelos grupos envolviam um grande volume de dados qualitativos, uma ênfase para esse tipo de análise foi dada. Orientou-se um método a partir da teoria fundamentada nos dados, e nesse sentido a análise dos dados seria baseada na ideia de codificação, cujo processo consiste em identificar conceitos (ou códigos) e categorias e avaliar na perspectiva do fenômeno de interesse. Posteriormente as relações entre as categorias deveriam ser analisadas para formar as proposições da teoria explícitas em causas e efeitos, condições e estratégia de ação. A Figura 2 ilustra um exemplo de um padrão recorrente identificado e analisado.

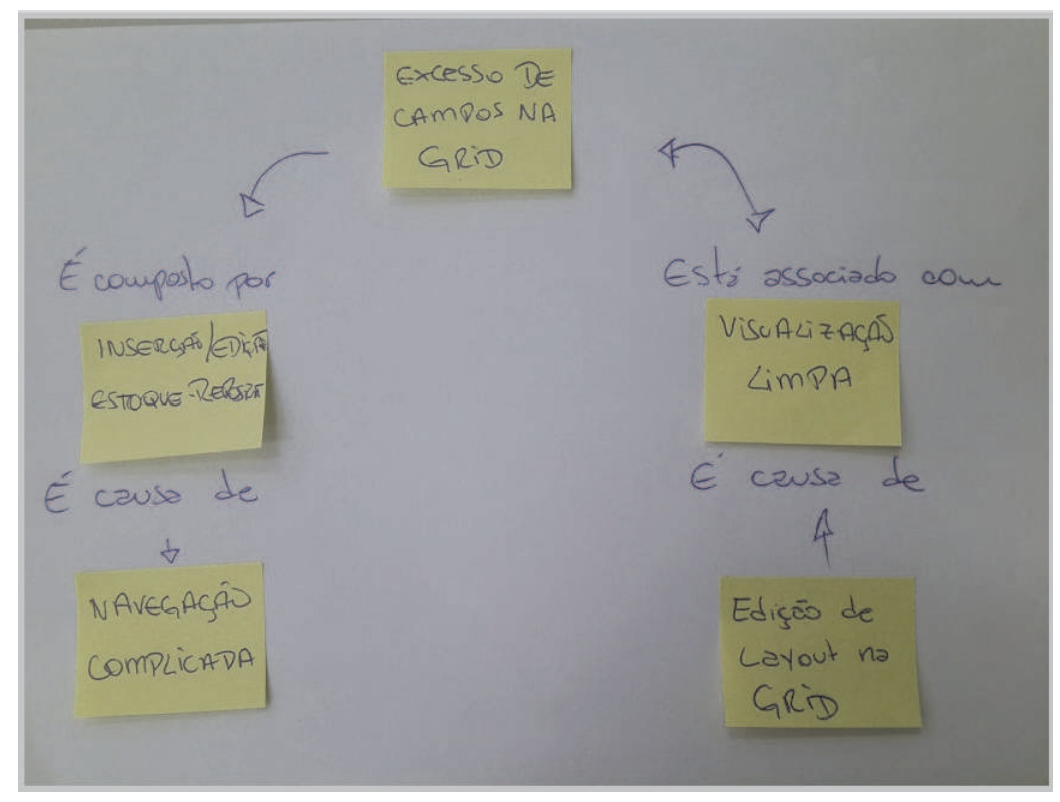

Figura 2. Padrão recorrente identificado

$\mathrm{Na}$ aplicação da técnica personas os grupos definiram uma persona para (1) caracterizar o usuário típico da aplicação; (2) determinar seus objetivos e a forma de interagir através da interface do módulo do ERP; (3) enumerar e visualizar o fluxo de execução das principais tarefas; e (4) perceber as dificuldades e as alternativas adotadas para transpor 
problemas encontrados ou necessidades não atendidas pelo sistema. A Figura 3 apresenta uma das personas criadas por um dos grupos para caracterizar o usuário típico do módulo em estudo.

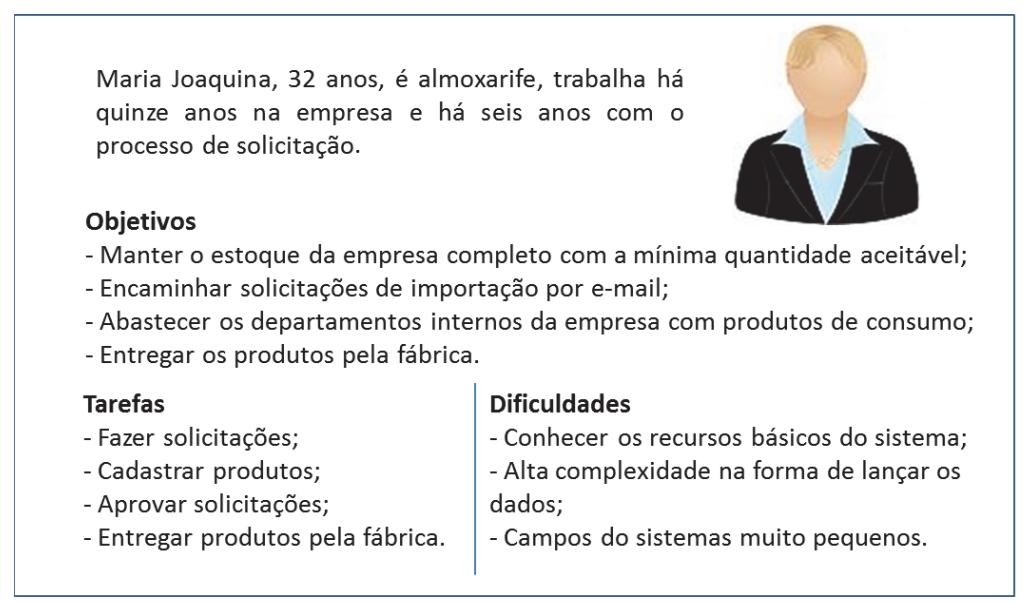

Figura 3. Persona caracterizando o usuário típico da aplicação

No quarto encontro, quinta etapa do workshop, aconteceu apresentação dos grupos dos resultados consolidados da coleta de dados e as personas criadas.

\subsection{Análise dos resultados do estudo de caso}

Observou-se que a metodologia proposta teve boa aceitação por parte dos colaboradores da empresa. Um questionário foi empregado com o intuito de capturar a visão dos participantes sobre as técnicas de coletas aprendidas e empregadas.

$\mathrm{Na}$ questão que solicitava classificar o conhecimento anterior ao workshop referente à técnica utilizada, 7 participantes $(64 \%)$ que escolheram a técnica de entrevista informaram ter um conhecimento médio da técnica. Esta questão revelou que o card sorting era a técnica de coleta de dados menos conhecida entre os participantes.

Os resultados das questões para classificar o grau de dificuldade que os participantes tiveram no uso das técnicas nas etapas de contatar o cliente, planejar o uso, visitar o cliente e analisar os dados coletados podem ser visualizados na Figura 4. Em cada uma das etapas, o grau de dificuldade foi considerado baixo para a maioria das técnicas utilizadas, sendo que as técnicas de investigação contextual e card sorting foram consideradas, pela maioria dos participantes que as escolheram, de grau médio de dificuldade no planejamento de uso, e o card sorting considerado de grau alto de dificuldade na etapa de contatar o cliente.

Na sequência uma questão foi elaborada com um conjunto de itens para medir o grau de concordância e o nível de aceitação das técnicas com base no Modelo de Aceitação de Tecnologia (TAM) [Davis 1989]. O modelo utilizado permite identificar fatores intrínsecos e extrínsecos, envolvidos nas decisões, intenção e satisfação dos indivíduos quanto à aceitação e ao uso da tecnologia da informação [Dias et al. 2011]. Os itens da questão foram elaborados e agrupados para análise conforme os fatores (1) percepção sobre facilidade de uso, (2) percepção sobre utilidade da técnica, e (3) percepção sobre facilidade de compreender. A escala Likert foi utilizada para que os entrevistados indicassem o grau de concordância ou discordância com cada uma das nove afirmações e 
para cada técnica utilizada. A escala tinha seis categorias de resposta que variavam de "discordo totalmente" a "concordo totalmente".

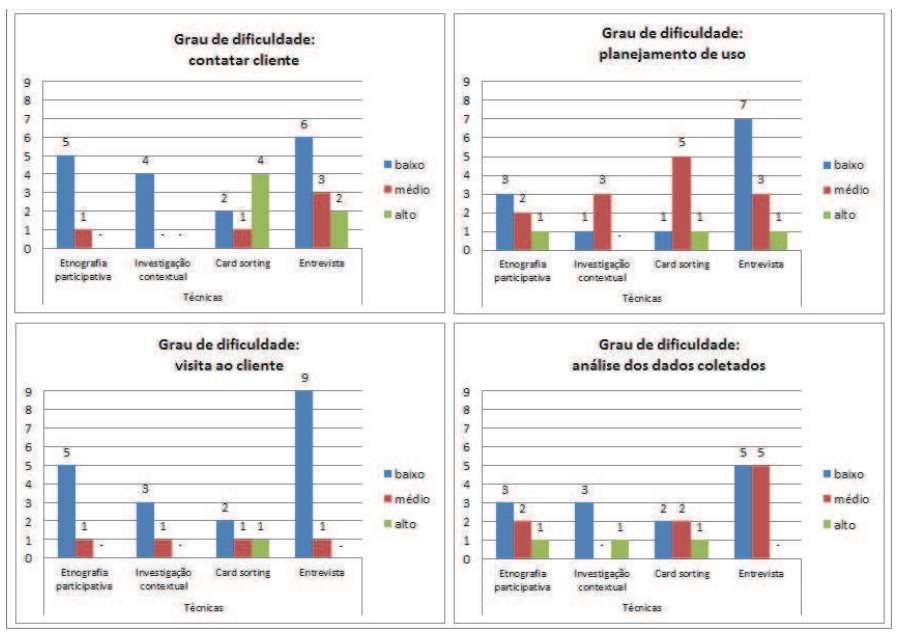

Figura 4. Grau de dificuldade referente às demandas da metodologia
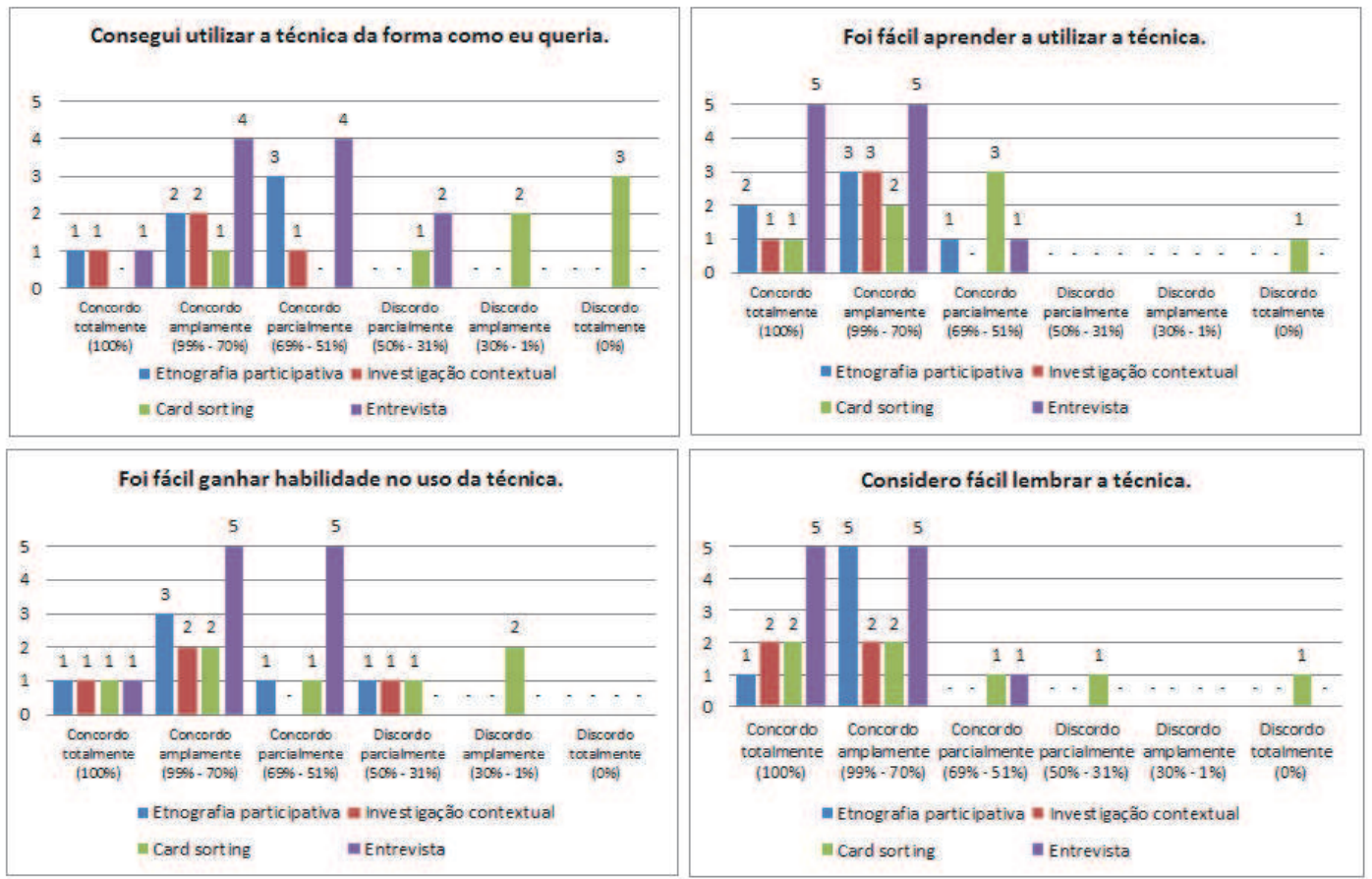

Figura 5. Percepção sobre a facilidade de uso

Nas afirmações para medir a percepção sobre facilidade de uso, os participantes deveriam indicar o quanto conseguiram utilizar a técnica da forma que queriam e o quanto foi fácil aprender a utilizar, ganhar habilidade e lembrar a técnica (Figura 5). A técnica da entrevista se destaca como a técnica mais fácil de usar.

Os itens para medir a percepção sobre a utilidade da técnica levaram os participantes a refletirem sobre o quanto a técnica utilizada permitiu descobrir problemas e novas 
necessidades de interação do usuário com o produto, além de revelar se usar a técnica melhorou a percepção sobre as necessidades de interação do usuário e se a técnica é útil para a coleta de dados. No primeiro e no segundo gráfico da Figura 6, se pode constatar que a maioria dos participantes concordam totalmente com as afirmações de que a técnica permitiu descobrir problemas e novas necessidades de interação do usuário com o produto.
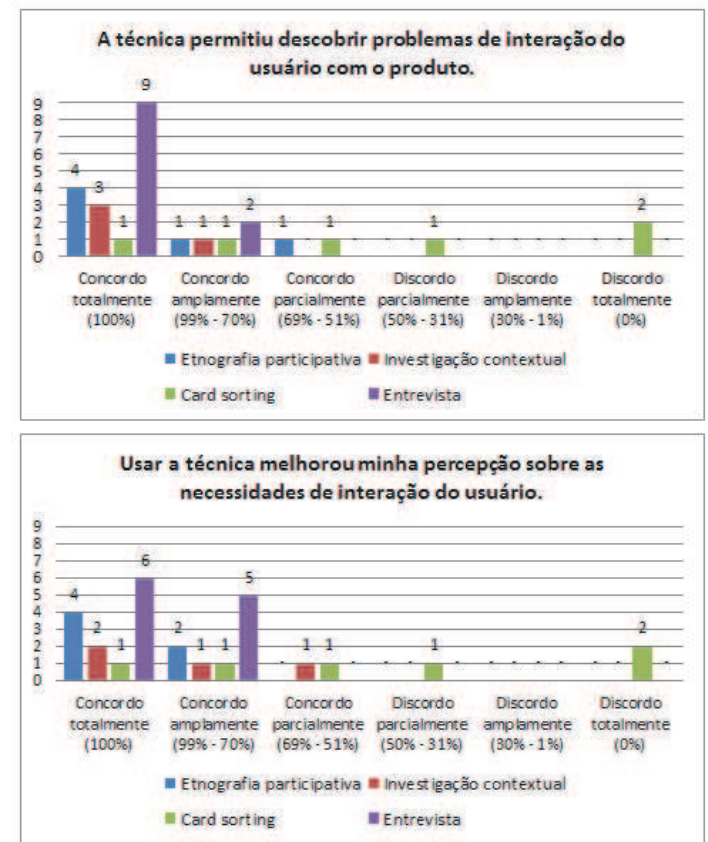

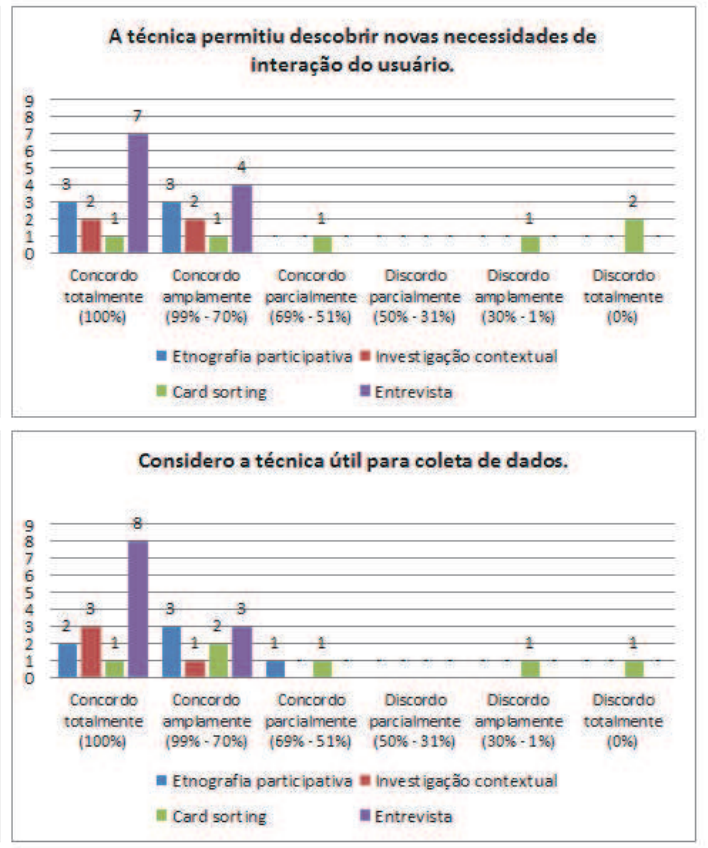

Figura 6. Percepção sobre a utilidade da técnica

O resultado sobre o quanto o planejamento da técnica e sua aplicação são fáceis de compreender pode ser visualizado na Figura 7.

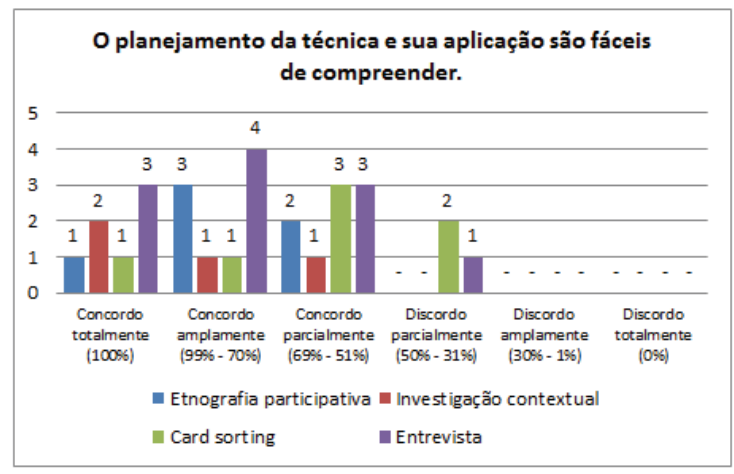

Figura 7. Percepção sobre a facilidade de compreender

Para calcular o somatório e as médias entre os participantes, em cada um dos itens e para cada técnica de coleta utilizada, atribuiu-se um valor numérico de 1 a 6 às categorias de resposta. Os valores foram atribuídos conforme a porcentagem de concondância, sendo 
que a concordância total (100\%) receberia maior escore (6) e a discordância total (0\%) o menor escore (1).

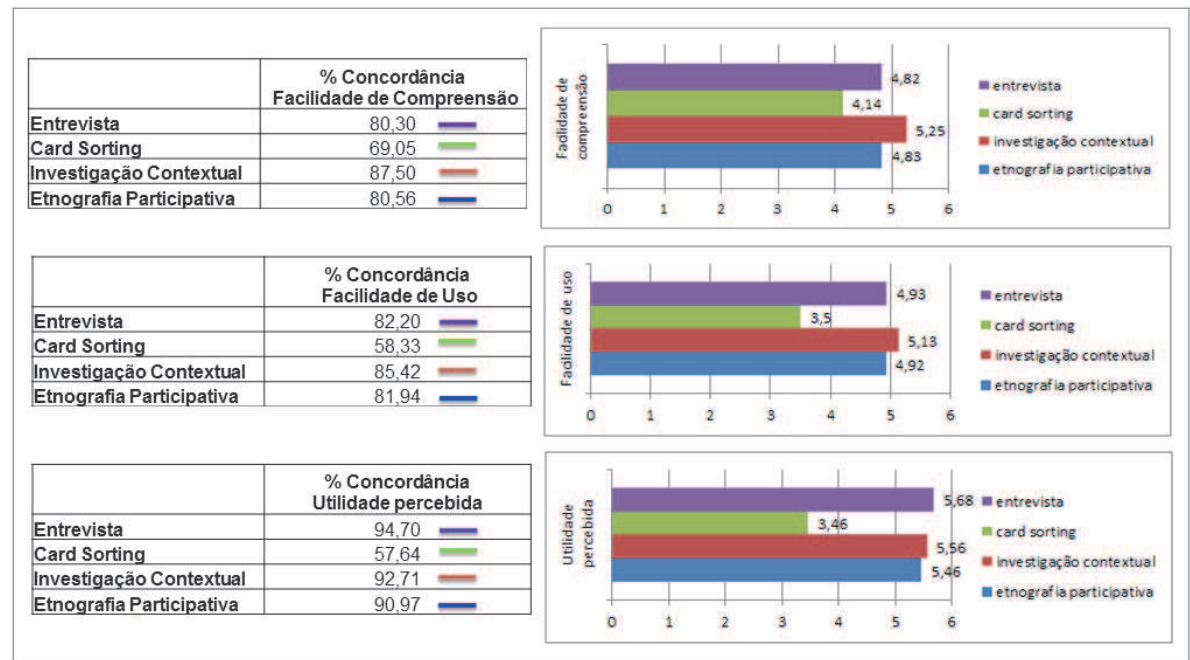

Figura 8. Análise do grau de concordância e o nível de aceitação das técnicas

A Figura 8 permite visualizar um resumo dos resultados e concluir que as menores porcentagens de concordância sobre utilidade percebida, facilidade de uso e facilidade de compreensão foram apontadas na técnica card sorting, contudo, o resultado não revelou discordâncias e nenhuma técnica foi rejeitada mantendo-se um bom nível de aceitação entre elas.

\section{Considerações finais}

A metodologia investigativa para identificar as necessidades de usuários aplicada atingiu seu principal objetivo que foi proporcionar uma vivência à equipe de desenvolvimento na prática de coleta de dados junto aos usuários de sistemas ERP. Durante a etapa de apresentação dos resultados os participantes teceram comentários que deixou evidente a percepção de como as técnicas de coleta bem planejadas e controladas são eficientes para extrair o que são de fato necessidades do usuário e que muitas vezes estão fora da visão dos desenvolvedores.

Além disso, a experiência relatada no estudo de caso foi fundamental para dar sequência ao plano de implantação da cultura de design da interação dentro da empresa, e demonstrar aos colaboradores a importância de utilizar o design da interação para a melhoria da aceitação, da produtividade e uso do ERP e impactar na satisfação de usuários finais.

\section{Agradecimentos}

Agradecemos a empresa Mega Sistemas Corporativos pelo apoio financeiro para o desenvolvimento do projeto relacionado a este trabalho.

\section{Referências}

Chou, H.-W., Chang, H.-H., Lin, Y.-H., and Chou, S.-B. (2014). Drivers and effects of post-implementation learning on ERP usage. Computers in Human Behavior, 35(0):267-277. 
Courage, C. and Baxter, K. (2005). Understanding your users: A practical guide to user requirements methods, tools, and techniques. Morgan Kaufmann.

Davis, F. D. (1989). Perceived usefulness, perceived ease of use, and user acceptance of information technology. MIS quarterly, pages 319-340.

Dias, G. A., da Silva, P. M., Delfino Jr, J. a. B., and de Almeida, J. R. (2011). Technology Acceptance Model (TAM): avaliando a aceitação tecnológica do open journal systems (OJS). Informação \& Sociedade: Estudos, 21(2).

Faisal, C. M. N., Tariq, S., Ahtram, T., Abbasi, M. S., Sarwer, S., and Selamat, A. (2012). A novel usability matrix for ERP systems using heuristic approach. In Management of e-Commerce and e-Government (ICMeCG), 2012 International Conference on, pages 291-296. IEEE.

Glaser, B. G. (1992). Emergence vs forcing: Basics of grounded theory analysis. Sociology Press.

Iivari, N. (2006). Representing the user in software development - a cultural analysis of usability work in the product development context. Interacting with Computers, 18(4):635-664.

Marshall, R., Cook, S., Mitchell, V., Summerskill, S., Haines, V., Maguire, M., Sims, R., Gyi, D., and Case, K. (2013). Design and evaluation: end users, user datasets and personas. Applied ergonomics.

Matthews, D. (2008). Usability as an ERP selection criteria. IFS White Paper, January.

Nunes, A. C., Alves, C. F., and Pinto, R. C. (2012). Modelagem de processos no apoio à aderência de implantação de ERP hospitalar. In SBSI 2012. VIII Simpósio Brasileiro de Sistemas de Informação., pages 231-242.

Padilha, T. C. C. and Marins, F. A. S. (2005). Sistemas ERP: características, custos e tendências. Revista Produção, 15(1):102-113.

Rogers, Y., Sharp, H., and Preece, J. (2013). Design de Interação - Além da Interação Humano Computador. Porto Alegre: Bookman.

Schylström, M. (2013). User experience in ERP system development: An action research project to involve user experience in the everyday work.

Scott, J. E. (2008). Technology acceptance and ERP documentation usability. Communications of the ACM, 51(11):121-124.

Seffah, A., Gulliksen, J., and Desmarais, M. C. (2005). An introduction to humancentered software engineering. In Human-Centered Software Engineering - Integrating Usability in the Software Development Lifecycle, pages 3-14. Springer.

Singh, A. and Wesson, J. (2009). Evaluation criteria for assessing the usability of ERP systems. In Proceedings of the 2009 annual research conference of the South African Institute of Computer Scientists and Information Technologists, pages 87-95. ACM.

Uflacker, M. and Busse, D. (2007). Complexity in enterprise applications vs. simplicity in user experience. In Human-Computer Interaction. HCI Applications and Services, pages $778-787$. Springer. 